\title{
Simulating impact of over-grazing on grassland degradation using GIS: a case study in the Heihe River Basin, China
}

\author{
Bingyu Wang ${ }^{\text {a }}$, Takashi Oguchi ${ }^{\text {a, b }}$, Lin Zhang ${ }^{c}$ \\ a Department of Natural Environmental Studies, Graduate School of Frontier Sciences, The University of Tokyo, Japan. \\ bingyu_ice@hotmail.com,oguchi@csis.u-tokyo.ac.jp \\ ${ }^{\mathrm{b}}$ Center for Spatial Information Science, The University of Tokyo, Japan. \\ 'Northwest Institute of Ecology and Environmental Resources, Chinese Academy of Sciences, China. zhanglingky@lzb.ac.cn
}

Keywords: over-grazing, grassland degradation, simulation

\begin{abstract}
:
Inland river basins in arid to semi-arid regions are widely distributed in Northwest China, Central Asia, Central Australia, and North Africa, and are often subject to significant human activities. The most distinctive natural feature of such basins is the shortage of water resources, and the pivotal reasons involve less precipitation and heavy evapotranspiration (ET). In recent years, intensive human activities also damage the natural environment of the basins. They result in many problems especially the deterioration of ecological environment which will lead to severe consequences such as desertification, sandstorm, the disappearance of wetlands, reduction of forest and grassland degradation. They prevent us from achieving the goal of sustainable development. How to balance economic development and ecosystem conservation and to realize the sense of sustainability in inland river basins will be vitally important.
\end{abstract}

The Heihe River is the second largest inland river in the Northwest of China with a long history development by human (Figure 1). Water resources from the river are crucial not only for the ecosystem but also for local human societies. The Heihe River Basin (HRB) is divided into three zones with different landscapes and natural environments. The upstream of HRB is the headstream which generates water resources mainly from glaciers and snow in Qilian Mountain. A large population of nomadic national minorities inhabits here and keeps animal husbandry as the primary production activity. In the early times, the Chinese government encouraged production activities to stimulate economic growth, and significant over-grazing and resultant severe grassland degradation occurred. Grassland is crucial for maintaining water resources especially in arid regions, without grasses most water will quickly evaporate into the air. Therefore, land resource management about grassland and the impact of human activities on the natural environment are of high research value in the HRB.
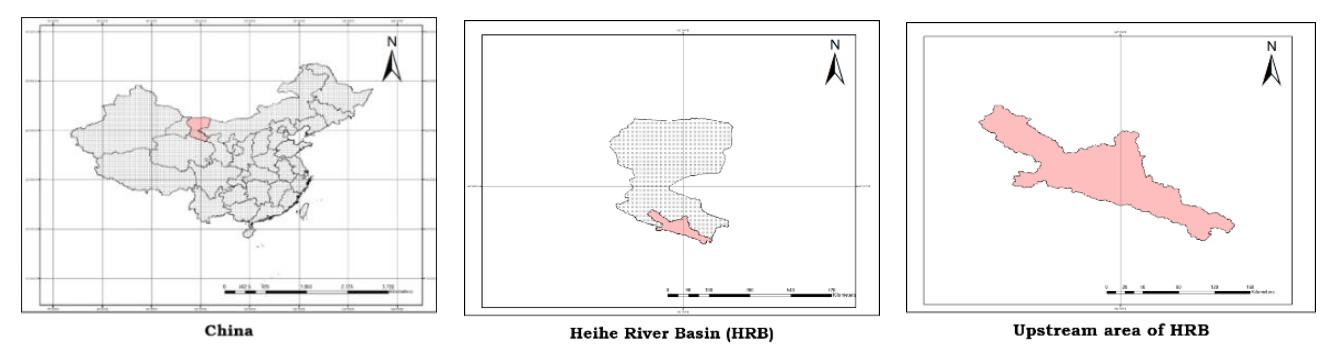

Figure 1. Geographic location of the study area

This research aims to investigate the impact of over-grazing on grassland degradation in the inland ecosystem of the HRB. The changes of grassland distribution were simulated under different over-grazing scenarios to provide a reference for resource management and the related decision-making process and to contribute to the sustainable development of the region.

Geographic Information Systems (GIS) were mainly applied for data processing and spatial analysis. The land use data for the Heihe upper stream area in 2011 was selected as the underlying map (Wang et al., 2014) and was reclassified with two types, grassland and non-grassland (Figure 2a). A DEM (Digital Elevation Model) and land use data for the study area (Figure 2b) were utilized to generate a seasonal pasture map (Figure 2c) associated with land use data. Also, this research integrates a grazing simulation model with a land use model to investigate the impact of overgrazing on grassland resource. The agent-based model (ABM) was applied for simulating the grazing processes with 
NetLogo as the primary interface mainly to generate grassland change quantity under various over-grazing scenarios. The land use model of Dyna-CLUE (Dynamic Conversion of Land-Use and its Effects model) was adopted to examine the spatial distribution of grassland degradation. Data collection and processing works were conducted including lab programming and field surveys.

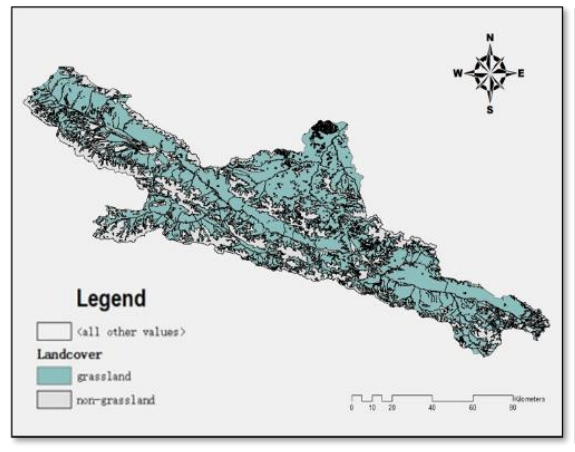

(a)

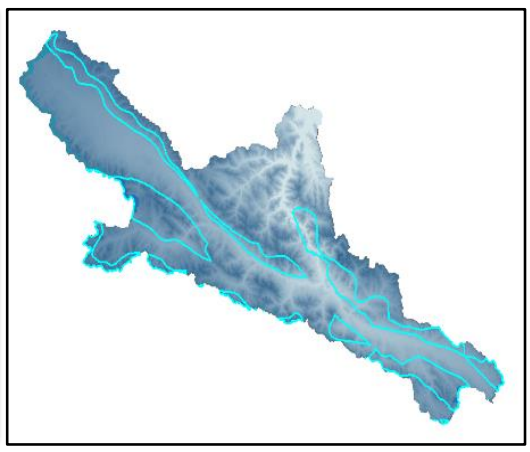

(b)

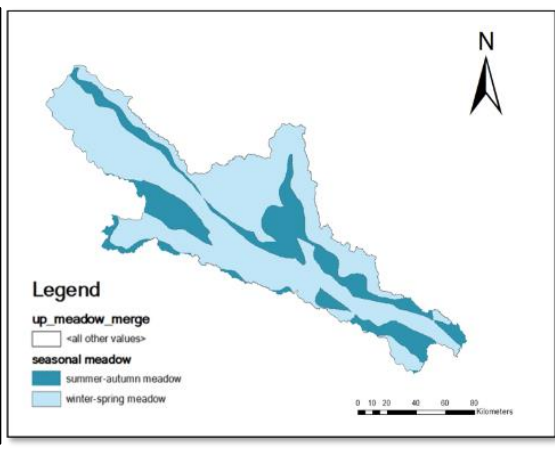

(c)

Figure 2. Maps for the upstream area of HRB (a) Land use reclassification map for the year 2011, (b) Elevation distribution based on the DEM (Li et al., 2010a,b, 2011), (c) Seasonal pasture map

Various experiments were conducted to develop a grazing model that can maximumly reflect the real conditions. After a series of test and verification, the model was confirmed to be capable of predicting the degradation of grasslands in future scenarios. The results show that over-grazing capacity and grazing patterns will simultaneously result in different levels of grassland degradation. With the increase of grazing capacity, the area of grassland degradation increases. The nomadism grazing pattern contributes more to grassland degradation than the fence grazing pattern.

Simulation results from ABM were coupled with the Dyna-CLUE model to generate the spatial distribution of grassland degradation (Figure 3). The resultant land use maps with six types (construction land, cultivated land, forest, grassland, unutilized land and water) provide a holistic viewpoint of land use management with future sceneries (Figure 3a). Also, this research emphasized the transformation between grassland degradation and grassland increment and the distribution of the variation (Figure 3b). The results show that grassland degradation tends to occur in the junction areas of the seasonal meadows and the boundary areas of HRB. This study has revealed the current problems that cause grassland degradation. Also, recommendations regarding sustainable development have been suggested to support the decision-making for grassland reservation and grazing management from a geospatial viewpoint.

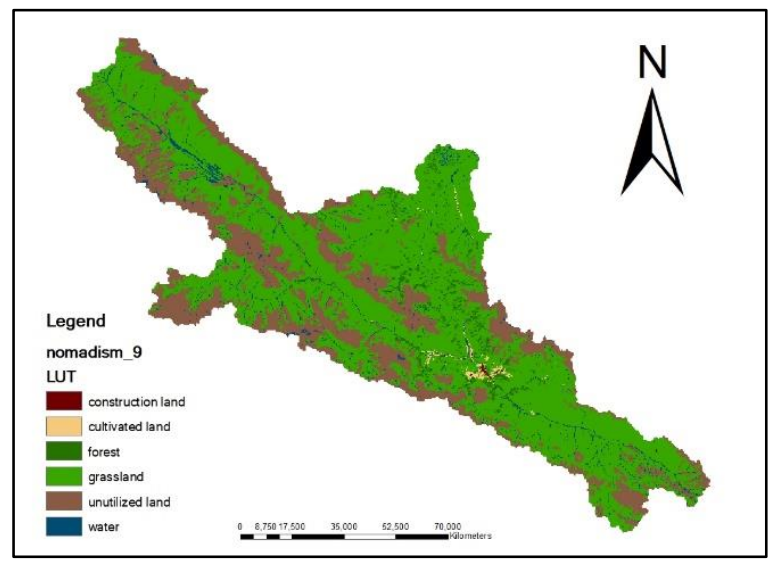

(a)

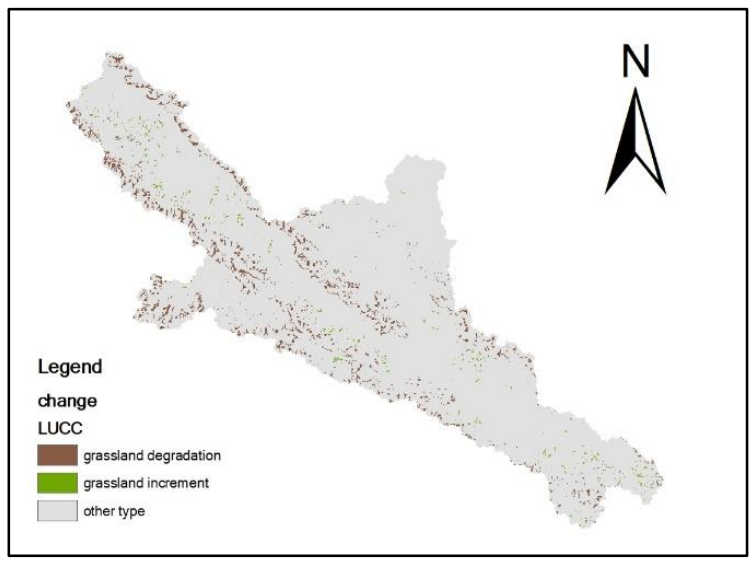

(b)

Figure 3. Prediction result of grassland degradation under certain over-grazing scenarios in the upstream area of HRB in 2025 (a) Land use map with six land types, (b) Grassland and degraded land variation map 